\title{
La industria brasileña y la absorción de conocimientos: determinantes internos y externos de la empresa ${ }^{1}$
}

\author{
Philipe Scherrer Mendes, Gustavo Britto y Ana Maria Hermeto
}

\section{Resumen}

La inversión en investigación y desarrollo (l+D), infraestructura y tecnología constituye una alternativa posible para los países en desarrollo que desean acelerar su trayectoria de crecimiento. En el caso del Brasil, reducir la brecha tecnológica requiere modificar la estructura productiva y aumentar la productividad, mediante la endogenización del progreso tecnológico. Definir los factores que determinan la inversión en absorción de conocimiento externo es fundamental. Para ello, se realizó un análisis multinivel basado en microdatos de la Pesquisa Industrial Anual-Unidade Local y la Pesquisa de Inovação-PINTEC (2008, 2011 y 2014) del Instituto Brasileño de Geografía y Estadística (IBGE). Los resultados sugieren que los determinantes internos de las actividades innovadoras de las empresas, se comportan conforme a la teoría. Los determinantes municipales serían coherentes con la literatura solo para los gastos en "máquinas y equipos" y "capacitación", mientras se observa la falta de influencia del espacio diversificado en la determinación de los gastos en I+D.

\section{Palabras clave}

Industria, empresas industriales, inversiones, innovaciones tecnológicas, conocimientos tecnológicos, cambio tecnológico, desarrollo industrial, Brasil

\section{Clasificación JEL \\ 014, R11}

\section{Autores}

Philipe Scherrer Mendes es Doctor en Economía de la Universidad Federal de Minas Gerais, Brasil. Correo electrónico: philipescherrer@gmail.com.

Gustavo Britto es Profesor Asociado en el Departamento de Ciencias Económicas de la Universidad Federal de Minas Gerais. Correo electrónico: gustavo@cedeplar.ufmg.br.

Ana Maria Hermeto es Profesora Asociada en el Departamento de Ciencias Económicas de la Universidad Federal de Minas Gerais. Correo electrónico: ahermeto@cedeplar.ufmg.br.

\footnotetext{
Los autores agradecen al Instituto Brasileño de Geografía y Estadística (IBGE) por el acceso a los microdatos y al Instituto de Investigación Económica Aplicada (IPEA) por el apoyo en la sala de datos confidenciales del IBGE. Se agradece, asimismo, el apoyo financiero del Consejo Nacional de Desarrollo Científico y Tecnológico (CNPq) del Brasil y de la Fundación de Apoyo a la Investigación del Estado de Minas Gerais (FAPEMIG).
} 


\section{Introducción}

Uno de los aspectos centrales del desarrollo económico se relaciona con el proceso de innovación, que de acuerdo con Schumpeter (1982) sería el único fenómeno capaz de aumentar la riqueza de una economía a largo plazo. En el debate sobre los procesos que conducen a la innovación, la producción, la adopción y la difusión de la innovación tecnológica son factores esenciales para el crecimiento económico y el cambio social. Asimismo, la innovación tecnológica es una característica distintiva de los productos y los sectores en los cuales los países compiten con éxito en el mercado mundial.

Los países con mayor diversidad productiva y composición tecnológica tienden a diferenciarse de los países cuya estructura de producción se basa en productos con bajos niveles de transformación o contenido tecnológico, que compiten por precio. Esta diferencia se ha utilizado para justificar la existencia de desequilibrios estructurales en la balanza comercial de los países en desarrollo, como consecuencia de la desigual generación y difusión del progreso tecnológico (Kaldor, 1957; Thirlwall, 2019; McCombie y Thirlwall, 1994, entre otros). La brecha tecnológica entre los países ha creado graves problemas de competitividad internacional, de manera que el crecimiento de las exportaciones de los países en desarrollo (que fabrican productos de bajo contenido tecnológico) está totalmente vinculado al comportamiento de la demanda internacional.

Los países industrializados tratan de crear condiciones para mantenerse siempre en la frontera del progreso tecnológico, disminuyendo la vulnerabilidad de su estructura de importaciones y exportaciones al exportar productos con mayor elasticidad ingreso y disminuir su importación. La industrialización por sí sola no parece capaz de disminuir la vulnerabilidad de las economías a las restricciones externas (Tavares, 1998). De ahí la importancia de la frontera tecnológica y de la endogenización de un proceso de avance e innovación constantes. Fajnzylber (1990) atribuye a los países rezagados la incapacidad de abrir la "caja negra" del progreso tecnológico y la falta de condiciones para aumentar sus niveles de producción y productividad.

La innovación en los países periféricos presenta peculiaridades que los distinguen de los países con industrias desarrolladas. Entendiendo el desarrollo económico como un proceso histórico, es importante considerar los aspectos socioeconómicos de la estructura de los países en desarrollo según el argumento de que el desarrollo consiste en la transición de una tecnología avanzada en una economía rezagada, que es incapaz de producirla de manera endógena como resultado de su proceso evolutivo (Merhav, 1972). En este sentido, invertir en la adquisición de conocimientos puede constituir una alternativa para el crecimiento. Sin embargo, esta adquisición no debe entenderse como una simple compra. Para que haya reales posibilidades de adquisición, las empresas deben invertir en la capacidad de absorber los conocimientos internamente. Esta capacidad sería un factor fundamental en la diferenciación de las empresas. Cohen y Levinthal (1990) definen la capacidad de absorción como la habilidad de reconocer el valor de la información externa, asimilarla y aplicarla con fines comerciales, haciendo hincapié en la importancia de la acumulación previa de conocimientos. Las fuentes externas serían fundamentales para el proceso de innovación y la capacidad de explorar esos conocimientos, evaluarlos y utilizarlos es un componente vital de la capacidad de una empresa para generar innovación.

Al definir al sector industrial como mayor generador de dinamismo y conectar los posibles desdoblamientos de esas inversiones en términos de productividad y crecimiento, es posible establecer un paralelismo entre el objetivo innovador de las empresas, que apunta a los beneficios y la participación en el mercado, y el resultado agregado de este proceso, desde la perspectiva del aumento de los niveles de productividad industrial y la importancia de este crecimiento para el desempeño económico general. En este sentido, el objetivo de este trabajo es medir, para la industria de transformación brasileña, los determinantes de la inversión en capacidad de absorción de las empresas industriales brasileñas, controlando la influencia ejercida por el territorio. 
El análisis contribuye con la literatura al analizar, conjuntamente, los determinantes internos y externos de la empresa en la determinación de las inversiones en absorción de conocimientos. El nivel de desagregación de los datos, la representatividad de las dos bases de datos utilizadas -la Pesquisa Industrial Anual-PIA (encuesta industrial anual) y la Pesquisa de Inovação-PINTEC (encuesta de innovación) del Instituto Brasileño de Geografía y Estadística (IBGE) - y el control de la heterogeneidad espacial, realizado con indicadores del mercado de trabajo formal, en un país con la dimensión territorial del Brasil y sus diferencias internas, refuerzan la importancia del análisis, sus resultados y conclusiones ${ }^{2}$. Otro aporte consiste en la diferenciación de dos tipos de inversiones en la adquisición de conocimientos, que se presentan como potenciales generadores de innovaciones y tienen diferentes interrelaciones con las características territoriales.

\section{Aspectos teóricos de la capacidad de absorción tecnológica}

\section{Importancia del desarrollo de esas capacidades}

Los estudiosos de los procesos que conducen a la innovación reconocen que la producción, la adopción y la difusión de la innovación tecnológica son esenciales para el desarrollo económico y el cambio social (Malerba, 1992; Teece y Pisano, 1994; Bell y Pavitt, 1997, entre otros) y que las innovaciones tecnológicas son una característica distintiva de los productos y sectores en los cuales los países con altos ingresos compiten con éxito en el mercado mundial. Así, la magnitud de la capacidad de absorción de una empresa (micro) se entrelaza con el perfil de la industria en que se inserta (meso) y la capacidad de generación de riqueza de un país (macro). Esto evidencia la importancia de esta literatura para la comprensión del desarrollo en su conjunto.

Las actividades innovadoras, como las que se describen a continuación, desempeñan un papel importante en la creación de capacidades tecnológicas, pues permiten una mejor comprensión de las direcciones que está tomando el mercado y la optimización de las inversiones hacia las oportunidades creadas. Malerba (1992) destaca que la actividad tecnológica de las empresas sería responsable de la creación de conocimientos fundamentales para el proceso de aprendizaje mediante la acción, el uso, la búsqueda y la interacción, entre otros. Desde una perspectiva similar, Bell y Pavitt (1997) destacan la importancia de la construcción de capacidades que permitan la generación y gestión de cambios tecnológicos, en particular la creación de habilidades productivas y la acumulación de conocimientos y experiencias.

Las decisiones que determinan las trayectorias de las empresas y su acumulación de conocimientos y habilidades permiten la construcción de un conjunto prácticamente exclusivo de capacidades para realizar sus actividades (Teece y Pisano, 1994). Sin embargo, el desafío consiste en crear condiciones propicias para la inversión en conocimientos y actividades innovadoras que permitan obtener los resultados esperados al caminar en la frontera tecnológica o cerca de ella. Si bien este es un problema que enfrentan las empresas establecidas en los países desarrollados, la situación se vuelve más compleja cuando el debate se traslada a los países de industrialización tardía. La existencia de acumulación en el proceso de progreso tecnológico permite que las empresas que se encuentran en la frontera tecnológica tengan ventajas con respecto a las que están atrasadas. Y es precisamente la búsqueda de este beneficio diferencial la que impulsa el progreso tecnológico.

\footnotetext{
2 De acuerdo con la Relação Anual de Informações Sociais (RAIS) (relación anual de informaciones sociales) del Ministerio de Economía.
} 
La velocidad con que se manifiesta el avance tecnológico depende de una serie de factores y existe una necesidad mínima de capacidad dentro de la empresa para que esta no sea expulsada del mercado ${ }^{3}$. Independientemente de los aspectos relacionados con el dinamismo sectorial, es necesario que las empresas conozcan el ambiente en el que operan para que, como mínimo, no se alejen de la frontera de producción al punto de ser expulsadas del mercado. Si la empresa no tiene suficiente dinamismo y acumulación de conocimientos para dictar las reglas que definen un sector, debe tener al menos la capacidad de comprender sus características para poder mantenerse en el mercado.

Cohen y Levinthal (1990) acuñaron el concepto de capacidad de absorción y lo definieron como la habilidad de reconocer el valor de la información externa, asimilarla y aplicarla con fines comerciales, que es, en gran medida, una función del nivel de conocimiento previo que la empresa posee. Las fuentes externas son fundamentales para el proceso de innovación en sí, por lo que la capacidad de explotar esos conocimientos es un componente vital de la capacidad de una empresa para generar innovación.

Al examinar la interacción entre universidades y empresas, Meyer-Krahmer y Schmoch (1998) destacan la importancia de la creación de capacidad de absorción, pues esta permite la percepción e inserción de las empresas en los nuevos paradigmas tecnológicos. Van den Bosh, Volberda y de Boer (1999) señalan que las empresas deben construir capacidad de absorción de conocimientos, porque esta permite agilizar los avances tecnológicos y puede significar un diferencial con respecto a los competidores. La existencia de habilidades que permitan a las empresas integrar, construir y reconfigurar competencias para reaccionar rápidamente a los cambios de contexto o paradigma es fundamental para su desempeño (Teece, Pisano y Shuen, 1997). De acuerdo con Zahra y George (2002), la capacidad de absorción debe entenderse como un conjunto de rutinas y procesos organizativos que permiten la creación de condiciones favorables para los avances productivos. Esto permitiría a las empresas la creación de aptitudes y habilidades para hacer frente a los cambios y las reconfiguraciones en sus actividades, permitiendo la obtención y el mantenimiento de ventajas competitivas. Históricamente las empresas que más invierten en capacidad de absorción aumentan las posibilidades de conquistar mayores oportunidades.

La capacidad de absorción supone un tipo de aprendizaje que no se parece al aprendizaje mediante la acción, porque no es un proceso automático en el cual se adquiere cada vez más práctica y eficiencia en una misma actividad. Si bien las empresas tienen diferentes formas de acumulación de conocimientos (procesos de aprendizaje), la generación de estas reservas y capacidades tecnológicas traería beneficios en las trayectorias tecnológicas y no solo en términos de costo de producción (Malerba, 1992). Se trata de un proceso costoso, que requiere esfuerzo y disciplina y genera ventajas tecnológicas para las empresas en la creación de condiciones favorables para la interiorización de conocimientos. Sin embargo, no se trata de algo que se crea de la noche a la mañana o con una solución simple y a corto plazo.

Desde la perspectiva de buscar caminos para optimizar la construcción de capacidad de absorción de conocimientos, Cohen y Levinthal (1989) encuentran un fuerte paralelismo entre esta capacidad y la inversión en investigación y desarrollo (I+D). Las empresas no solo invierten en I+D para buscar directamente nuevos procesos o productos, sino también para desarrollar y ampliar sus capacidades para asimilar y explotar la información disponible externamente. Así, el incentivo a la absorción derivada de los desbordamientos sería mayor en los sectores en que las dificultades de aprendizaje son mayores. Además, el ambiente de aprendizaje afecta el impacto de los desbordamientos en los gastos en investigación y desarrollo y la importancia de ampliar la capacidad de absorción -en relación con las condiciones negativas de apropiabilidad- está condicionada por el grado de interdependencia de los competidores.

\footnotetext{
3 Factores como oportunidades tecnológicas, paradigmas tecnológicos, entre otros.
} 
La definición de los factores determinantes de la construcción de las habilidades es fundamental para optimizar las trayectorias y crear condiciones que minimicen la brecha tecnológica. Este es el desafío que debe buscarse en las economías de industrialización tardía, entendiendo el progreso industrial como determinante del crecimiento económico del país, a partir del aumento de los niveles de productividad de las empresas, la mejora de los procesos, los avances en términos de complejidad productiva y el incremento de la elasticidad ingreso de los productos. Esto permite una mejor inserción en el comercio internacional y, por medio de la demanda agregada, el aumento de los niveles de ingreso y la creación de una mayor dinámica productiva, incrementando los niveles de productividad y, por consiguiente, la remuneración del trabajo.

La adquisición de tecnologías y conocimientos externos permite, pero no garantiza, la mejora del desempeño tecnológico del país importador. Desde esta perspectiva, si bien es posible que las naciones más rezagadas mejoren sus fronteras con respecto a las naciones avanzadas, se necesita una capacidad mínima que permita la absorción y el uso efectivos del conocimiento adquirido. En este sentido, se debe realizar una labor de capacitación tecnológica constante, con el fin de ampliar las aptitudes tecnológicas que permiten un mejor uso de las tecnologías importadas (Chiarini, 2014).

La innovación tecnológica debe entenderse como un proceso de aprendizaje en el que las innovaciones no son necesariamente radicales, sino que existe la posibilidad de que pequeñas innovaciones incrementales contribuyan a aumentar la productividad de la empresa o el país (Rosenberg, 1993). El aprendizaje descrito por Abramovitz (1986) sería posible por la existencia de un diferencial de etapas de desarrollo tecnológico de los países, en que el desempeño de los países seguidores depende de sus condiciones iniciales. El desarrollo de "habilidades sociales" sería un factor crucial para crear las condiciones para la determinación y absorción del conocimiento tecnológico existente, que al ser apropiado por los seguidores tendería a aumentar sus niveles de productividad. Esta "habilidad social" sería una competencia técnica que no puede comprenderse sin tener en cuenta otros importantes actores o instituciones políticas, comerciales, industriales y financieras, entre otras, además de los niveles educativos y la estructura organizativa e institucional en la cual se insertan las empresas. Para Abramovitz (1986), la combinación de la brecha tecnológica y la "habilidad social" determinaría el potencial de algunos países para promover su convergencia, mejorando sus niveles de productividad. Para que eso ocurra es necesario que existan canales para promover los flujos de conocimiento, desde los líderes hacia los seguidores.

Algunas economías de industrialización tardía pasaron de ser pobres y tecnológicamente atrasadas a ser prósperas y relativamente modernas, con la creación de un importante conjunto de empresas industriales, fabricantes de productos tecnológicamente complejos, que compiten de manera eficaz con los productos de las empresas establecidas en los países avanzados (Kim y Nelson, 2005). La obtención y el progresivo control de nuevas tecnologías ha sido un aspecto central de las economías de industrialización tardía que crecieron rápidamente después de la década de 1980. Además de una fuerte inversión en capital físico y humano, es fundamental destacar la adquisición y asimilación de tecnologías existentes en los países avanzados, que en conjunto transformaron a algunos países considerados imitadores en la década de 1960 en innovadores en la década de 1990. Sin embargo, este no es un proceso simple o con rápido retorno. El hecho de que el conocimiento tecnológico no esté distribuido en forma equitativa entre las empresas y no pueda imitarse o transferirse fácilmente entre ellas hace que esta transferencia requiera necesariamente un significativo nivel de capacidad de absorción, pues sus principios no son fácilmente asimilables.

Dentro del paradigma desarrollista-industrial, la búsqueda de la superación de las condiciones de subdesarrollo requiere, necesariamente, la superación de la dependencia tecnológica y la creación de condiciones favorables para endogenizar la generación de tecnología, incluso aunque esto requiera, en un primer momento, la adopción de técnicas extranjeras con el fin de acelerar el proceso. Esta adopción de técnicas extranjeras o importación de tecnología no debe considerarse como un fin, sino 
como una oportunidad para la creación interna de condiciones para el futuro desarrollo de nuevas formas de producir o nuevos productos. Es importante tener cautela con esta simple importación, pues puede llevar a las economías atrasadas a permanecer en la trampa de la dependencia tecnológica. Las tecnologías fácilmente disponibles serían tecnologías maduras, cuyo dinamismo tecnológico se ha agotado. Aunque superiores con respecto a la tecnología nacional, se estarían alejando de la frontera tecnológica y favorecerían el mantenimiento de la distancia tecnológica entre los países (Chiarini, 2014).

Un aspecto importante que debe considerarse en este debate es que las condiciones propicias para la difusión y absorción del conocimiento están limitadas geográficamente. Así, comprender la manera en que la localización de las empresas se relaciona con la creación de condiciones para la absorción de conocimientos también es un objetivo de este trabajo. En la próxima sección se aborda el debate sobre la dinámica del progreso tecnológico desde una perspectiva regional, presentando y discutiendo las características territoriales que dinamizan la innovación y la producción.

\section{Condicionantes territoriales}

En la literatura sobre economías de aglomeración se presenta la relación entre el crecimiento industrial y la productividad de las empresas, que derivan de las economías externas de escala, condicionada por la estructura productiva de la región. La proximidad geográfica, elemento fundamental en la determinación de la actividad innovadora y el progreso tecnológico, permite a las empresas intercambiar información y avanzar tecnológicamente. Esto contribuye a mitigar la incertidumbre, característica inherente a la actividad innovadora (Feldman, 1994), y a crear una externalidad productiva que reduce los costos de los nuevos descubrimientos. De este modo, los desbordamientos tecnológicos tienden a concentrarse en el espacio, lo que justifica la aglomeración productiva en busca de estos beneficios. A grandes rasgos, es posible distinguir dos corrientes teóricas que sustentan este debate. Si bien los pensamientos de estas dos corrientes no son excluyentes, existe una clara diferenciación entre Marshall (1982) y Jacobs (1975). Para Marshall (1982), esas externalidades provienen de la especialización de la actividad industrial y pueden sintetizarse de la siguiente manera: efectos de encadenamiento intersectoriales-proveedores-usuarios, desbordamientos tecnológicos de conocimiento entre empresas y beneficios por la formación de polos de trabajo especializados. Para Jacobs (1975), la principal fuente de externalidades de que pueden disfrutar las empresas es la diversidad de actividades económicas desarrolladas en las ciudades. La multiplicidad de bienes y servicios, tecnologías y conocimientos propios de un centro urbano diversificado potencia el intercambio de ideas (cross fertilization of ideas) (Glaeser y otros, 1992). En otras palabras, las innovaciones se originan a partir de la fecundación de ideas entre los diversos sectores de actividades instalados en una misma ciudad y conducidos por la generación de nuevos tipos de trabajo, lo que aumenta la capacidad de generación de nuevos bienes y servicios.

Las ventajas de la aglomeración urbana no se limitan al ámbito de la producción. Las grandes ciudades ofrecen una mayor variedad de bienes de consumo y servicios públicos y mayores posibilidades de contactos sociales, que se traducirían en externalidades. Así, estas también se vuelven atractivas para los trabajadores y consumidores. La disposición de las actividades productivas en el espacio también sufre la influencia de factores disgregantes. Sin embargo, estos factores no deben entenderse como la inexistencia o no manifestación de factores aglomerantes. En otras palabras, los beneficios de la aglomeración siguen siendo válidos para todos los actores del proceso productivo al mismo tiempo en que actúan los factores disgregantes. No obstante, solo aquellos actores que logran superar las dificultades derivadas de las fuerzas centrífugas tienden a ocupar las mejores posiciones o lugares centrales. El principal factor disgregante estaría relacionado con el ingreso de la tierra o ingreso del suelo urbano, que deriva de la existencia del derecho de propiedad. Este supone la necesidad de una remuneración por el uso del lugar (alquiler por unidad de superficie) y tiende a diferenciar los valores de los alquileres de acuerdo con su localización y proximidad con respecto al mercado consumidor 
(Von Thünen, 1966). La dinámica de precios elevados en lugares centrales y precios más baratos a medida que aumenta la distancia determina el tipo de actividad productiva que tiende a localizarse en cada lugar. La capacidad de pagar esta diferencia de alquiler está asociada a la rentabilidad de cada actividad, de manera que solo las más rentables (beneficio por unidad de superficie) logran acceder a los espacios centrales. De este modo, la determinación de la localización de la producción deriva de un gradiente de productividad, que genera una jerarquía espacial, y el ingreso del suelo urbano ejerce un poder disgregante porque expulsa a aquellos que no pueden pagar para instalarse en lugares privilegiados.

Glaeser y otros (1992) sugieren que los desbordamientos tecnológicos tienen lugar entre empresas de una misma industria y afectan positivamente el crecimiento. Los autores sostienen que la especialización productiva facilita el acceso a insumos, bienes y servicios intermedios, mano de obra y mercados. Los defensores de la existencia de una externalidad derivada de la especialización productiva cobraron importancia en la teoría económica regional y realizaron diversos trabajos empíricos para evaluar la adhesión de esta teoría a la realidad (Glaeser y otros, 1992; Combes, 2000; Henderson, 2003, entre otros). A pesar de su respaldo empírico, demostrado en varios trabajos, es notoria la existencia de ciudades o regiones con una significativa dinámica económica que no constituyen un espacio especializado. En esta laguna, Jacobs (1975) presenta otra importante caracterización del espacio como factor generador de dinamismo. Sin negar la eficiencia productiva proporcionada por la especialización, Jacobs (1975) entiende que las posibilidades generadas por los espacios especializados son relativamente limitadas en comparación con las que se crean en los espacios diversificados. La diversificación productiva sería responsable en gran medida del florecimiento de nuevas ideas y conocimientos. Si bien la importancia de la región y del contacto cara a cara es similar a la adjudicada por Marshall (1982), sería la posibilidad de complementariedad o del cruce de información y tecnologías diferentes en diversos sectores la principal responsable del surgimiento de un mayor dinamismo económico. Así, las regiones con una gran variedad de sectores productivos tendrían mejores probabilidades de innovación, sobre todo en virtud de las posibilidades creadas por el intercambio y la recombinación de conocimientos y prácticas (Jacobs, 1975).

Como sugieren Duranton y Puga (2000), la diversificación y la especialización pueden coexistir con la tendencia a que las grandes ciudades sean más diversificadas y estables desde el punto de vista del tamaño, y lo mismo ocurre con las actividades presentes en ellas. Mientras la mayoría de las innovaciones suele ocurrir en ciudades diversificadas, al igual que el surgimiento de nuevas plantas productivas, las ciudades especializadas son el principal destino de la reubicación de las empresas que salen de las ciudades diversificadas. Sobre este último punto, la justificación presentada es que, una vez que las empresas encuentran su proceso de producción ideal, no necesariamente tienen más incentivos para permanecer en las ciudades diversificadas, de manera que compensan los elevados costos de producción con los beneficios derivados de la localización y tienden a trasladarse a otros lugares donde otras empresas comparten su especialización.

La búsqueda constante de nuevos productos y nichos de mercado promovió la dispersión geográfica de la producción, buscando, en la ruptura con la rigidez productiva del período fordista, una mayor flexibilidad en los mercados de trabajo, con nuevas formas de suministro de insumos y servicios que demandaron una serie de transformaciones en los patrones organizativos y comerciales. De acuerdo con Ernst y Kim (2002), las grandes corporaciones mundiales (empresas multinacionales) serían protagonistas de este nuevo modelo, al maximizar la utilización de los recursos dispersos a nivel mundial, accediendo a competencias localizadas en distintos lugares, cuya complementariedad permite una mayor eficiencia productiva. Mientras la actividad productiva se dispersaba en el territorio, surgieron nuevas formas de centralización territorial, generalmente relacionadas con la gestión de alto nivel de las operaciones y su control central. Estos movimientos presentan una reorganización marcadamente impulsada por los factores disgregantes relacionados con el costo de la localización. En este contexto, las empresas más intensivas en mano de obra tienden a disgregarse primero (Puga y Venables, 1996), 
afectadas por el aumento de los niveles salariales en las regiones desarrolladas, dando lugar a la creación de nuevas centralidades, poseedoras de masa crítica productiva.

Los avances tecnológicos han determinado que el costo de separar la actividad productiva sea cada vez más bajo, creando fuertes incentivos para que las ciudades cambien su especialización de una dimensión sectorial a una dimensión basada en las funciones desempeñadas. Dado que la misma decisión es tomada por un gran número de empresas, esta termina por definir el patrón de empleo de las ciudades y da lugar a ciudades industriales y ciudades que alojan los centros de actividades básicas de las empresas ${ }^{4}$. Esta nueva división aportaría mayores beneficios de proximidad a las actividades básicas que a las actividades productivas. Esta constatación lleva a la observación de que las ciudades que alojan el núcleo de las empresas son mucho más grandes y su número es más reducido, mientras las ciudades industriales son más numerosas y más pequeñas (Duranton y Puga, 2000).

Barbour y Markusen (2007) señalan que las actividades de innovación y desarrollo suelen estar localizadas en las regiones de origen de la empresa, mientras las actividades más rutinarias de producción y servicios estarían dispersas en lugares donde los costos son más bajos y regiones con un bajo patrón de consumo. Los autores destacan la importancia del análisis ocupacional, en detrimento del análisis sectorial, y hacen hincapié en que las empresas de un mismo sector, en función de esta nueva realidad en su estructura productiva, pueden presentar marcos ocupacionales totalmente diferentes en distintos lugares, lo que sería determinante del crecimiento de la productividad y el desempeño regional.

\section{Peculiaridades del caso brasileño}

Al llevar esta discusión al Brasil, un país con un desarrollo industrial tardío y una significativa heterogeneidad espacial, es posible encontrar características territoriales más o menos favorables para la difusión del conocimiento tecnológico ${ }^{5}$. La carencia de una capacidad social mínima que permita la absorción de conocimiento (Abramovitz, 1986) determina la exclusión de gran parte del territorio brasileño del progreso productivo e industrial. A pesar de ello, es indiscutible que el país experimentó una reorganización de la estructura productiva y la distribución espacial después de la década de 1990. Esta reestructuración, más conectada con el mercado externo y más demandante de mano de obra calificada, comenzó a sufrir una mayor influencia de los factores de localización. En este contexto, la urbanización se presentó más claramente como un elemento importante de la nueva dinámica de reestructuración productiva. En el marco de este proceso, recientemente han cobrado importancia nuevos estudios sobre las economías de aglomeración, que relacionan las economías externas de escala y la estructura productiva regional con los niveles de productividad (Galinari, Lemos y Amaral, 2006; Fontes, Simões y Oliveira, 2010; Freitas, 2012).

El crecimiento económico, con distribución del ingreso, registrado en el Brasil en la década de 2000 estuvo fuertemente influenciado por la demanda externa de productos básicos, que creó una dinámica interna con las consecuentes incorporación de mano de obra y generación de ingresos. La crisis internacional de 2008 parece haber cambiado un poco el curso de la historia: la economía brasileña perdió impulso en ese proceso y el crecimiento económico acompañado por el aumento del empleo y del ingreso sufrió un fuerte impacto. El agotamiento del modelo económico brasileño de la década de 2000 pone nuevamente de relieve la importancia de examinar los factores que determinan el aumento de los niveles de productividad ${ }^{6}$. En este debate, el papel desempeñado por la industria

\footnotetext{
4 Sede de la empresa, que comprende las actividades de gestión, los servicios comerciales y el centro de investigación y desarrollo, entre otros.

5 La estructura productiva e industrial brasileña se concentra fuertemente en el estado de São Paulo y en la región Sudeste, a pesar de las recientes políticas que fomentaron la redistribución regional de la industria, especialmente hacia el Nordeste.

6 Aumento de los precios de los productos básicos, entrada de capitales, expansión del consumo, bajas tasas de ahorro, entre otros factores.
} 
es innegable (Messa, 2015), tanto por su mayor intensidad de capital, como por ser una importante fuente de innovaciones y por su capacidad de generar empleos de mejor calidad, remuneración y menor rotación, favoreciendo el desarrollo de un capital humano específico. Cavalcante, Jacinto y De Negri (2015) señalan la importancia de aumentar los niveles de productividad de la economía brasileña para reanudar el ciclo de crecimiento económico. En el debate sobre el crecimiento de la productividad, los autores destacan las inversiones en investigación y desarrollo e innovación como motores de la productividad del trabajo futura. En ese mismo trabajo los autores ponen a prueba la hipótesis de que la productividad del trabajo en los sectores de menor intensidad tecnológica sería más sensible a la adquisición de máquinas y equipos que a la inversión en investigación y desarrollo y encuentran el resultado que la confirma.

Así como el proceso de innovación sufre una considerable influencia de su dimensión local, con el paso de los años la capacidad de absorción de conocimientos de la industria brasileña tiende a sufrir una mayor influencia de factores relacionados con la proximidad. De esta manera, se entiende que las empresas localizadas en regiones especializadas tienden a gastar más en la adquisición de máquinas y equipos y la capacitación de la mano de obra, en busca de mejores prácticas productivas, mientras el desempeño de las empresas localizadas en regiones diversificadas dependería de su capacidad de gozar de los beneficios de la pluralidad de este espacio localizado que, además de una mayor oferta de mano de obra calificada, promueve el florecimiento de la actividad innovadora relacionado con el proceso de investigación y desarrollo.

En este sentido, se estiman los determinantes de la capacidad de absorción de conocimientos de la industria de transformación brasileña, sobre la base de los microdatos de la PINTEC. El análisis se realiza para todas las empresas presentes en la PINTEC, en 2008, 2011 y 2014, y tiene en cuenta tanto los determinantes internos de la empresa como los municipales, en una estructura jerárquica de empresa y municipio. El período elegido corresponde a una importante reestructuración productiva nacional, marcado por las consecuencias de la crisis internacional de 2008.

\section{Base de datos y metodología}

El análisis empírico supone la combinación de distintas bases de datos. Las principales son: Pesquisa de Inovação-PINTEC, Pesquisa Industrial Anual-Empresa (PIA-Empresa), ambas del IBGE, y Relação Anual de Informações Sociais (RAIS) del Ministerio de Economía. Debido a la forma en que están organizadas las dos primeras bases de datos, con la identificación de las empresas por el Registro Nacional de Personas Jurídicas (CNPJ), se estructuró una base de datos en la que la unidad de observación es la empresa-unidad local y los datos de la RAIS están agregados a nivel municipal. En función de la periodicidad de la PINTEC, el análisis se realiza para 2008, 2011 y 2014. Cabe destacar que el análisis empírico se realiza con microdatos de la PINTEC y la PIA-Empresa, a los que es difícil acceder debido a la confidencialidad de la información.

\section{Metodología}

El análisis de las empresas industriales brasileñas desde la perspectiva de la inversión en actividades innovadoras evidencia la importancia de considerar el ambiente en el que estas se insertan. Asumiendo que este también es un factor condicionante de la trayectoria, el análisis empírico tiene en cuenta los controles para los efectos de la localidad (municipio). De esta forma, se decidió utilizar un modelo multinivel. Esta decisión se basa en las características que el análisis asume relacionadas con la interacción con el ambiente en el que se insertan las empresas, con la posible relación de causalidad mutua entre los 
individuos (en este caso las empresas) y el ambiente, interrelación que no puede ignorarse en el análisis (Goldstein, 1995). La posibilidad de una relación entre la variabilidad de las características individuales condicionadas al ambiente en el que se insertan las empresas debe considerarse en el análisis pues, aunque haya un control para las características individuales y del ambiente, existe la posibilidad de que -al no hacerse la distinción entre los niveles jerárquicos y su correlación - las estimaciones sean espurias. En el marco de esta perspectiva de análisis, con las empresas incluidas en grupos distintos, hay que suponer que sus características no son totalmente independientes del ambiente y que puede haber grupos que, en promedio, condicionan más o menos ciertas características de las empresas.

El modelo más simple de análisis jerárquico es el análisis de la varianza con efectos aleatorios. Esta primera especificación capta las diferencias entre la media general de la variable de respuesta y las medias específicas de cada grupo del segundo nivel y se representa conforme la siguiente especificación (Raudenbush y Bryk, 2002):

$$
\begin{gathered}
Y_{i j}=\beta_{0 j}+r_{i j} \\
\beta_{0 j}=\gamma_{00}+u_{0 j} \\
\gamma_{i j}=\gamma_{00}+u_{0 j}+r_{i j}
\end{gathered}
$$

con $Y_{i j}=$ variable dependiente para cada individuo $i$ en un grupo $j ; \beta_{0 j}=$ media de la variable dependiente para cada grupo $j ; r_{i j}=$ término de error del individuo $i$ en el grupo $j$ (normalmente distribuido con media 0 y varianza $\left.\sigma^{2}\right) ; \gamma_{00}=$ media poblacional de la variable dependiente; y $u_{0 j}=$ efecto aleatorio del grupo $j$ (o desviación del grupo $j$ de la media poblacional), teniendo $u_{0 j}$ una distribución normal. La varianza de la variable de respuesta estará dada por:

$$
\operatorname{Var}\left(Y_{i j}=\operatorname{Var}\left(u_{0 j}+r_{i j}\right)=\tau_{00}+\sigma^{2}\right.
$$

La descomposición de la varianza ( $\tau_{00}$-entre grupos y $\sigma^{2}$-dentro de los grupos) permite el cálculo del coeficiente de correlación intraclase, que indica la parte de la varianza total que se explica por el segundo nivel jerárquico, es decir en qué medida el ambiente condiciona el comportamiento individual. El coeficiente de correlación intraclase $(\mathrm{CCl})$ está representado por:

$$
\rho=\tau_{00} /\left(\tau_{00}+\sigma^{2}\right)
$$

Con un modelo genérico de análisis multinivel aplicado al análisis de la capacidad de absorción (CA), se obtiene?:

$$
C A_{i j}=\beta_{0 j}+\beta_{1 j}\left(1 \text { nivel }_{i j}\right)+r_{i j}
$$

con:

$$
\begin{gathered}
\beta_{0 j}=\gamma_{00}+\gamma_{01}\left(2 \text { nivel }_{j}\right)+u_{0 j} \\
\beta_{1 j}=\gamma_{10}
\end{gathered}
$$

Adaptando la propuesta de Cohen y Levinthal (1990) a las especificidades de la PINTEC, incluyendo variables explicativas del mercado de trabajo y la estructura urbana, se miden los determinantes del gasto en la creación de capacidad de absorción. La variable "capacidad de absorción" se construyó utilizando las variables de la actividad innovadora de la empresa. Conforme la PINTEC estas son: investigación y desarrollo internos, adquisición de investigación y desarrollo externos, adquisición de otros conocimientos externos, adquisición de software, adquisición de máquinas y equipos y capacitación ${ }^{8}$. De acuerdo con Araújo y Salerno (2015), es posible agrupar las actividades innovadoras en dos factores distintos, uno "basado en investigación y desarrollo", altamente correlacionado con la investigación

\footnotetext{
7 No se hizo la interacción entre las variables de primer y segundo nivel ni la aleatorización de las inclinaciones, de manera que la especificación de $\beta_{l j}$ es más simple.

8 Si bien las inversiones en "Introducción de innovaciones tecnológicas en el mercado" y "Otros preparativos para la producción y distribución" también son actividades innovadoras según la PINTEC (IBGE, s/f), estas dos inversiones no se abordaron porque no se relacionan con ninguna de las dos dimensiones de la innovación examinadas y presentadas en este trabajo.
} 
y el desarrollo internos y externos, y otro "basado en máquinas y capacitación", correlacionado con la adquisición de máquinas y equipos y la capacitación ${ }^{9}$. A partir del debate presentado en Araújo y Salerno (2015), las actividades innovadoras se dividen en dos grupos. El primero representaría un tipo de capacidad de absorción más cercano al concepto original de Cohen y Levinthal (1990), con una alta correlación del gasto en investigación y desarrollo, internos o externos, responsable de la generación de habilidades favorables al reconocimiento, la asimilación y la aplicación de información externa en las rutinas internas de las empresas. El segundo tipo, presentado como una posible alternativa a las economías rezagadas en su desarrollo económico e industrial, estaría relacionado con las inversiones en máquinas y equipos y capacitación, que permitirían la absorción y utilización de los conocimientos generados e incorporados en máquinas y equipos más modernos, suponiendo que tales inversiones tienden a realizarse para mejorar la eficiencia productiva.

A partir de la idea de actividades "basadas en investigación y desarrollo" y "basadas en máquinas y capacitación", se crearon dos grupos distintos de actividades innovadoras:

- La suma de los valores gastados en la investigación y el desarrollo internos, la adquisición de investigación y desarrollo externos y la adquisición de otros conocimientos, definida como "CA-producto"10; y

- La suma de los valores gastados en la adquisición de software y la adquisición de máquinas y equipos y capacitación, definida como "CA-proceso".

El primer grupo representaría un tipo de capacidad de absorción más propicia para el desarrollo de nuevos productos y el segundo grupo estaría correlacionado con la posibilidad de innovar en procesos productivos.

Una vez definida la construcción de las variables dependientes, fue necesario desarrollar una estrategia que permitiera evaluar los efectos de las inversiones en capacidad de absorción de conocimientos desde una perspectiva territorial. Esto se debe a que los datos de la PINTEC no incluyen una desagregación que permita evaluar la influencia ejercida por el territorio (en este caso, el municipio) ${ }^{11}$. Para ello, se establecieron dos estrategias que implicaron agregar los datos de la PINTEC a la información de localización de la empresa, mediante la PIA-Unidade Local (PIA-UL), conforme el Registro Nacional de Personas Jurídicas de la empresa. La primera estrategia consistió en utilizar las empresas de la PIA-UL que tenían solo una unidad local, excluyendo a las demás, y la segunda estrategia en generar una segunda base de datos con la división de los valores de la PINTEC (Registro Nacional de Personas Jurídicas) entre las diferentes unidades locales que tenían el mismo Registro ${ }^{12}$. En la próxima sección se presentan los resultados de las estimaciones econométricas para las dos estrategias.

En el cuadro 1 se presentan las variables utilizadas en los ejercicios empíricos y los dos niveles jerárquicos considerados en el análisis. El cuadro también incluye una pequeña descripción de cada una de las variables ${ }^{13}$.

9 Los autores realizan un análisis factorial de las actividades innovadoras de la PINTEC 2008 y obtienen dos factores que condensarían su importancia: uno altamente correlacionado con la investigación y el desarrollo internos y externos, que los autores denominan "innovación basada en investigación y desarrollo", y otro altamente correlacionado con la adquisición de máquinas y equipos y capacitación, que denominan "innovación basada en máquinas y capacitación".

${ }^{10}$ Las variables se deflactaron por el índice de precios al por mayor industrial (IPA-indústria) a precios de 2008, y se realizó la transformación logarítmica (In).

${ }^{11}$ La única desagregación territorial de la PINTEC corresponde a la unidad de la federación (IBGE, s/f).

${ }^{12}$ La información presente en la base de datos de la PIA-UL no permitió una mejor determinación de las unidades locales que habrían sido responsables de la ejecución de los gastos o donde estarían asignados los profesionales dedicados a investigación y desarrollo, entre otros datos recolectados por la PINTEC, por lo que se realizó una división simple, de manera que cada unidad local recibiera la misma parte de los gastos.

${ }^{13}$ Los indicadores de jerarquía tecnológica ocupacional se construyeron de acuerdo con Rodrigues, Oliveira y Albuquerque (2007) y los indicadores de intensidad tecnológica sectorial de acuerdo con Cavalcante (2014). 


\section{Cuadro 1}

Descripción de las variables utilizadas en el análisis empírico

\begin{tabular}{|c|c|c|}
\hline Nombre de la variable & Indicador & Construcción de la variable \\
\hline \multicolumn{3}{|c|}{ Primer nivel: características de la empresa } \\
\hline Ingresos netos & Escala productiva & Ln de los ingresos netos de ventas declarados en el balance de la empresa \\
\hline $\begin{array}{l}\text { Investigación y } \\
\text { desarrollo continuos }\end{array}$ & Oportunidad tecnológica & $\begin{array}{l}\text { Variable ficticia para empresas que declararon realizar actividades } \\
\text { de investigación y desarrollo de forma continua }\end{array}$ \\
\hline Mantener mercado & \multirow[t]{2}{*}{ Grado de apropiabilidad } & $\begin{array}{l}\text { Variable ficticia para empresas que consideraban muy importante realizar } \\
\text { innovaciones para mantener su participación en el mercado }\end{array}$ \\
\hline Ampliar mercado & & $\begin{array}{l}\text { Variable ficticia para empresas que consideraban muy importante realizar } \\
\text { innovaciones para ampliar su participación en el mercado }\end{array}$ \\
\hline Acuerdo de cooperación & Cooperación para innovar & $\begin{array}{l}\text { Variable ficticia para empresas que participaron en acuerdos de cooperación } \\
\text { con otras organizaciones }\end{array}$ \\
\hline $\begin{array}{l}\text { Doctores - dedicación } \\
\text { exclusiva }\end{array}$ & \multirow[t]{2}{*}{$\begin{array}{l}\text { Perfil de la mano de obra en } \\
\text { investigación y desarrollo }\end{array}$} & \multirow[t]{2}{*}{$\begin{array}{l}\text { Número de magísteres y doctores con dedicación exclusiva a la investigación } \\
\text { y el desarrollo }\end{array}$} \\
\hline $\begin{array}{l}\text { Magísteres - dedicación } \\
\text { exclusiva }\end{array}$ & & \\
\hline $\begin{array}{l}\text { Clasificación Nacional de } \\
\text { Actividades Económicas, } \\
\text { versión } 2.0 \\
\text { (CNAE 2.0) - División }\end{array}$ & Control sector industrial & Variables ficticias para los 24 sectores industriales - industria de transformación \\
\hline \multicolumn{3}{|c|}{ Segundo nivel: características municipales } \\
\hline Diversificación industrial & \multirow[t]{3}{*}{ Indicadores de urbanización } & Índice de diversificación industrial (Hirschman-Herfindahl modificado) \\
\hline $\begin{array}{l}\text { Distancia con respecto } \\
\text { a São Paulo }\end{array}$ & & Distancia con respecto a la ciudad de São Paulo, medida en horas \\
\hline $\begin{array}{l}\text { Concentración espacial - } \\
\text { servicios productivos }\end{array}$ & & $\begin{array}{l}\text { Índice de concentración espacial (QL) para servicios productivos (Relação Anual } \\
\text { de Informações Sociais (RAIS)) }\end{array}$ \\
\hline $\begin{array}{l}\text { Concentración espacial - } \\
\text { baja intensidad }\end{array}$ & \multirow[t]{4}{*}{$\begin{array}{l}\text { Indicadores de } \\
\text { especialización productiva }\end{array}$} & $\begin{array}{l}\text { Índice de concentración espacial (QL) para industrias de baja intensidad } \\
\text { tecnológica (RAIS) }\end{array}$ \\
\hline $\begin{array}{l}\text { Concentración espacial - } \\
\text { medio baja intensidad }\end{array}$ & & $\begin{array}{l}\text { Índice de concentración espacial (QL) para industrias de intensidad tecnológica } \\
\text { medio baja (RAIS) }\end{array}$ \\
\hline $\begin{array}{l}\text { Concentración espacial - } \\
\text { medio alta intensidad }\end{array}$ & & $\begin{array}{l}\text { Índice de concentración espacial (QL) para industrias de intensidad tecnológica } \\
\text { medio alta (RAIS) }\end{array}$ \\
\hline $\begin{array}{l}\text { Concentración espacial - } \\
\text { alta intensidad }\end{array}$ & & $\begin{array}{l}\text { Índice de concentración espacial (QL) para industrias de alta intensidad } \\
\text { tecnológica (RAIS) }\end{array}$ \\
\hline Ocupación - alta superior & \multirow[t]{6}{*}{$\begin{array}{l}\text { Indicadores de } \\
\text { mercado de trabajo }\end{array}$} & $\begin{array}{l}\text { Participación de empleados en ocupaciones de jerarquía tecnológica } \\
\text { "alta superior" (RAIS) }\end{array}$ \\
\hline Ocupación - alta inferior & & $\begin{array}{l}\text { Participación de empleados en ocupaciones de jerarquía tecnológica } \\
\text { "alta inferior" (RAIS) }\end{array}$ \\
\hline Ocupación - medio superior & & $\begin{array}{l}\text { Participación de empleados en ocupaciones de jerarquía tecnológica } \\
\text { "medio superior" (RAIS) }\end{array}$ \\
\hline $\begin{array}{l}\text { Educación - superior } \\
\text { completa }\end{array}$ & & Participación en la industria de empleados con educación superior completa (RAIS) \\
\hline Educación - maestría & & Participación en la industria de empleados con maestría (RAIS) \\
\hline Educación - doctorado & & Participación en la industria de empleados con doctorado (RAIS) \\
\hline \multicolumn{3}{|l|}{ Controles regionales y de año } \\
\hline Norte & \multirow{5}{*}{$\begin{array}{l}\text { Variables ficticias } \\
\text { - para controles de } \\
\text { características regionales }\end{array}$} & Variable ficticia Norte \\
\hline Nordeste & & Variable ficticia Nordeste \\
\hline Sudeste & & Variable ficticia Sudeste - Se omitió el estado de São Paulo \\
\hline Centro Oeste & & Variable ficticia Centro Oeste \\
\hline Sur & & Variable ficticia Sur \\
\hline 2011 & \multirow{2}{*}{$\begin{array}{l}\text { Variables ficticias para } 2011 \\
\text { y } 2014 \text { - } 2008 \text { omitida }\end{array}$} & Variable ficticia de 2011 \\
\hline 2014 & & Variable ficticia de 2014 \\
\hline
\end{tabular}

Fuente: Elaboración propia. 
El objetivo de la estimación de la capacidad de absorción, diferenciando entre la absorción más propicia para la innovación de productos y la absorción más propicia para la innovación de procesos, es verificar las posibles diferencias entre ambas, controlando para características de la empresa y observando la manera en que estas difieren en términos de los determinantes del nivel territorial, para poder relacionar las diferentes estructuras urbanas con las distintas actividades de absorción de conocimiento.

Se pone a prueba la hipótesis de que las actividades innovadores más intensivas en investigación y desarrollo - generadoras, por lo tanto, de capacidad de absorción de conocimientos propicios para la innovación de productos - presentan una fuerte correlación con la diversificación productiva (Jacobs, 1975; Duranton y Puga, 2001; Storper y Venables, 2004; Araújo, 2014, entre otros) y con los sectores de mayor intensidad tecnológica (Henderson, Kuncoro y Turner, 1995; Araújo, 2014, entre otros), y que las actividades innovadoras más intensivas en máquinas, equipos y capacitación - generadoras, por lo tanto, de capacidad de absorción de conocimientos propicios para la innovación de procesos - presentan una mayor correlación con la especialización productiva y con los sectores de menor intensidad tecnológica (Duranton y Puga, 2001; Barbour y Markusen, 2007; entre otros).

\section{Análisis de los resultados}

Se presentan y discuten los resultados relativos a las dos estrategias empíricas adoptadas, con estimaciones que tienen en cuenta solo a las empresas que tenían una única "unidad local", lo que no representó un sesgo en la perspectiva territorial pero supuso la exclusión de una parte significativa de empresas $^{14}$, y estimaciones que tienen en cuenta a todas las empresas presentes en la PINTEC, para lo que fue necesario dividir los valores gastados en actividades innovadoras entre las diferentes "unidades locales", como se mencionó anteriormente, con el consiguiente sesgo en la perspectiva territorial.

\section{Capacidad de absorción e innovación de productos}

Los resultados presentados en esta sección apoyan la discusión de las características de la empresa y del municipio más favorables a las inversiones en actividades innovadoras que lograron desarrollar las habilidades para reconocer, asimilar y utilizar los conocimientos tecnológicos existentes o disponibles en el proceso de innovación (CA-producto).

El primer paso para la estructuración del modelo jerárquico es la estimación del modelo incondicional (análisis de la varianza), que permite determinar la parte de la varianza total del modelo que se explica en el primer y el segundo nivel, siendo, por lo tanto, una medida de la influencia del ambiente (municipio) en el comportamiento individual (empresa). Junto con esta primera especificación, en el cuadro 2 se presentan los resultados de las estimaciones para los indicadores de la empresa (primer nivel) y para el modelo general, que también incluye controles municipales.

\footnotetext{
${ }^{14}$ Es posible suponer que las empresas más grandes fueron excluidas de este análisis.
} 


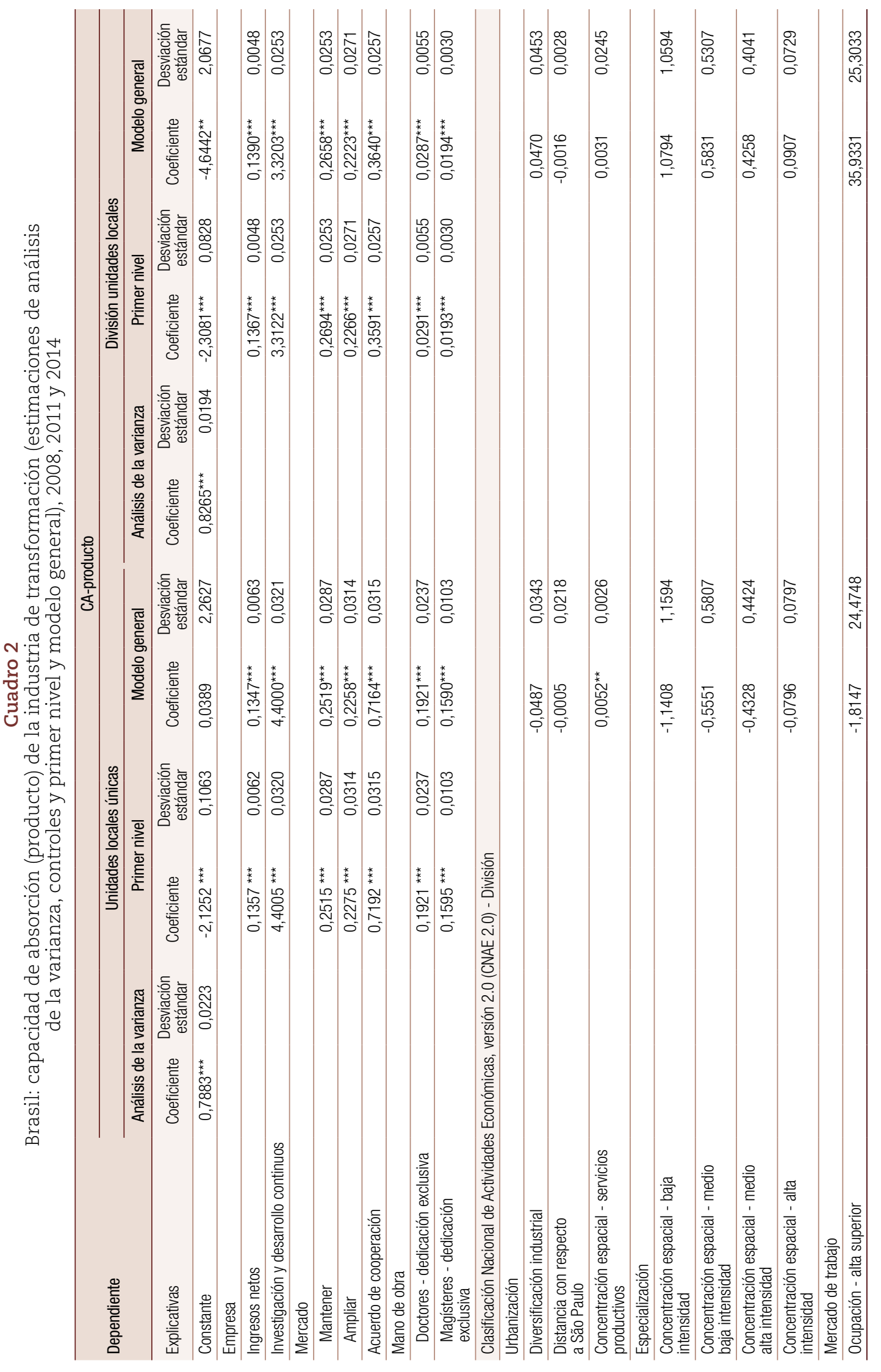




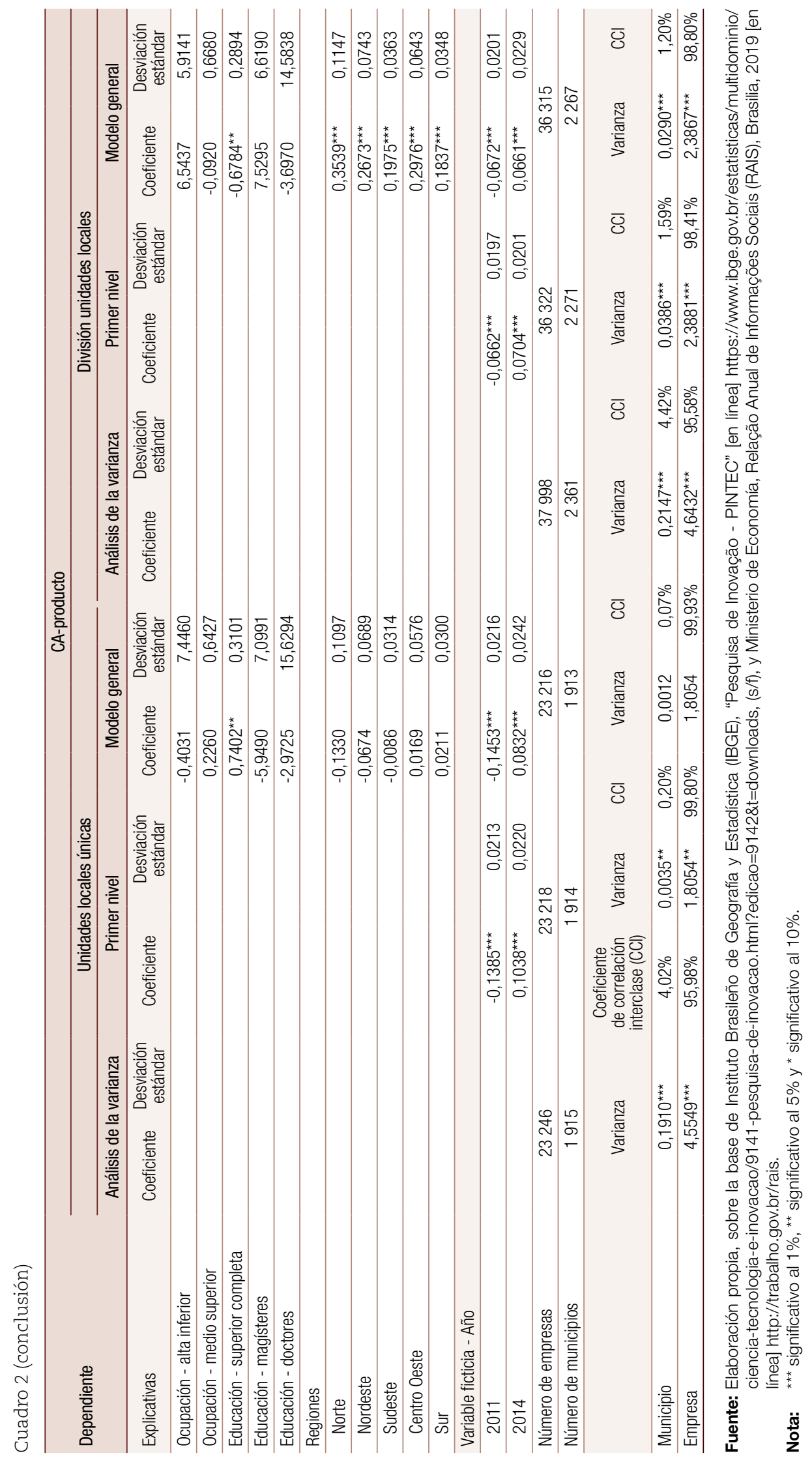


La estimación del análisis de la varianza justifica la utilización del modelo jerárquico, el coeficiente de correlación intraclase es significativo al $1 \%$ y los municipios responden por el 4,02\% y el 4,42\% de la varianza de los datos, para las dos bases de datos. Como se esperaba, la inclusión de variables explicativas de primer nivel reduce la participación municipal en la varianza total de los datos y la varianza sigue siendo significativa.

En la estimación de los condicionantes de primer nivel, se destaca que los resultados concuerdan con lo esperado para los dos grupos de empresas: el aumento de los ingresos netos (tamaño de la empresa) aumenta los gastos en "CA-producto"; las empresas que declararon realizar investigación y desarrollo de forma continua gastan más que las demás empresas; las empresas que consideraban muy importante realizar innovaciones para mantener o ampliar su participación en el mercado gastan más que las demás empresas; las empresas que participaron en algún acuerdo de cooperación para el desarrollo de actividades innovadoras gastan más que las demás; el aumento de la participación de magísteres y doctores dedicados exclusivamente a actividades de investigación y desarrollo aumenta los gastos en "CA-producto". Para este último resultado, se destaca la diferencia de magnitud entre las dos estimaciones, pues el resultado para las "unidades locales únicas" es bastante superior con respecto a la "división de los valores entre unidades locales". Esto sugiere una posible limitación de la estrategia empírica adoptada de dividir los valores equitativamente entre las unidades locales. También a nivel de la empresa, se incluyeron variables ficticias sectoriales (Clasificación Nacional de Actividades Económicas, versión 2.0 (CNAE 2.0)- División) y variables ficticias para 2011 y 2014, que mostraron que en 2011 los gastos fueron menores que en 2008 y que en 2014 fueron mayores.

Con la inclusión de variables de segundo nivel en el modelo general se procuraba filtrar todos los posibles efectos de la empresa y territoriales que serían responsables de la determinación de las inversiones en capacidad de absorción propicia para el desarrollo de productos. Los resultados para las variables de primer nivel se mantuvieron significativos y conforme lo esperado, como en las especificaciones anteriores. También se observó el mantenimiento de los resultados para los controles sectoriales y para las variables ficticias de año. En general, a pesar de algunos cambios en la significación estadística de los coeficientes de control de las características municipales y del cambio de signo en el caso de la variable de educación superior completa, los resultados obtenidos en las estimaciones de los determinantes de los gastos en "CA-producto" encuentran amparo teórico.

Como era de esperar, los controles para las variables de urbanización no fueron significativos en esta especificación general. La idea con dichos controles era verificar los efectos de la localización en la determinación de los gastos en capacidad de absorción de conocimientos, poniendo a prueba las hipótesis de Jacobs (1975) y Marshall (1982) y la literatura que aborda los desdoblamientos de estas dos propuestas originales para los gastos en actividades innovadoras relacionadas con la investigación y el desarrollo. Se esperaba que los determinantes municipales de los gastos en "CA-producto" estuvieran positivamente correlacionados con la urbanización, la diversificación productiva y los sectores de mayor intensidad tecnológica, pero esto no se observó. Solo la variable de servicios productivos para el modelo de "unidades locales únicas" fue significativa al $5 \%$. Los controles para especialización productiva no fueron significativos en ninguna de las dos estimaciones.

El único de los controles municipales incluidos que resultó significativo (al 5\%) fue el de participación municipal de empleados con educación superior completa en la industria. Sin embargo, este resultado presenta signos diferentes entre los dos grupos de empresas analizados. En el caso de la estimación de las empresas cuyos gastos se dividieron entre las unidades locales, los resultados sugieren que el aumento de la proporción de empleados con educación superior completa en los municipios disminuye los gastos en "CA-producto". Esto puede explicarse por la mayor dispersión territorial de los empleados con educación superior completa, que contrasta con la concentración espacial de los gastos en actividades relacionadas con la investigación y el desarrollo, además de que hubo un mayor crecimiento de la proporción de empleados con educación superior completa en los sectores de menor 
intensidad tecnológica, también menos intensivos en estos gastos. Por otra parte, las empresas con "unidades locales únicas" habrían logrado aprovechar este espacio y obtener mayores gastos en estas actividades innovadoras en los municipios con mayor participación de estos trabajadores.

\section{Capacidad de absorción e innovación de procesos}

Siguiendo procedimientos similares a los realizados en la sección anterior, los resultados presentados aquí sustentan la discusión de las características propicias para las inversiones en absorción de conocimientos externos, desde la perspectiva de que los países atrasados tienen la alternativa de absorber conocimientos productivos mediante la adquisición de mejores insumos productivos, máquinas y equipos, y mediante la capacitación de su mano de obra. Esas inversiones tienden a generar mejoras productivas al permitir nuevas formas de hacer y, así, a ser potenciales generadoras de innovaciones de procesos. Se evalúan los factores internos y de localización de la empresa que determinan los gastos en lo que en este trabajo se denomina "capacidad de absorción-proceso". Los resultados esperados son una menor dependencia de los efectos espaciales relacionados con la urbanización (proximidad a São Paulo, oferta de servicios productivos y diversificación industrial) y una mayor dependencia de los espacios especializados (coeficientes de localización (Ql) industriales) y con factores que influyen en la reducción de los costos de mano de obra (Puga y Venables, 1996; Duranton y Puga, 2001; Duranton y Puga, 2005; Barbour y Markusen, 2007; entre otros).

Una primera constatación que surge del cuadro 3 es que hay una disminución del coeficiente de correlación intraclase en comparación con el modelo de análisis de la varianza que midió los determinantes de las inversiones relacionadas con las actividades de investigación y desarrollo. Esto sugiere que, como se esperaba, las actividades e inversiones relacionadas con la investigación y el desarrollo son más sensibles a las influencias territoriales que los gastos en máquinas y equipos y capacitación.

El crecimiento de los ingresos netos influye positivamente en los gastos en "CA-proceso" en una magnitud superior a la ejercida en "CA-producto", ambos positivos y significativos; la importancia atribuida a las inversiones innovadoras como estrategia para ampliar o mantener su mercado es superior, y también significativa al 1\%, en la determinación de los gastos en "CA-proceso" en comparación con "CA-producto"; la continuidad de las actividades de investigación y desarrollo afecta positivamente los gastos en "CA-proceso", sin embargo menos que la influencia ejercida en "CA-producto"; la participación en acuerdos de cooperación con otras organizaciones aumenta los gastos en "CA-proceso"; la existencia de magísteres y doctores con dedicación exclusiva no influye significativamente en los gastos en "CA-proceso" para el análisis en que los gastos se dividieron entre las unidades locales, mientras los afecta significativamente en el análisis relativo a las "unidades locales únicas". En este caso el número de doctores con dedicación exclusiva aumenta los gastos en "CA-proceso" y el número de magísteres con dedicación exclusiva los disminuye. Las variables ficticias para 2011 y 2014 muestran que esos gastos sufrieron una reducción después de la crisis internacional de 2008, aunque el impacto haya sido mayor en 2011 en comparación con 2014. Con la inclusión de las variables de primer nivel el coeficiente de correlación intraclase disminuye y sigue siendo significativo al $1 \%$.

Los resultados del modelo general son coherentes con los obtenidos anteriormente y los controles de primer nivel mantienen signos, significaciones y magnitudes similares a las estimaciones únicamente de primer nivel. En el modelo general se observó una reducción de los gastos en "CA-proceso" con el crecimiento de la participación municipal de los trabajadores en ocupaciones de nivel jerárquico alto-superior y una reducción con el crecimiento de la participación de los trabajadores con educación superior completa, ambos relacionados con el perfil municipal del trabajador industrial y el incremento de los costos de la mano de obra. Los municipios con mayor participación de trabajadores en ocupaciones de jerarquía tecnológica medio superior influyeron positivamente en esos gastos de las empresas industriales brasileñas. 


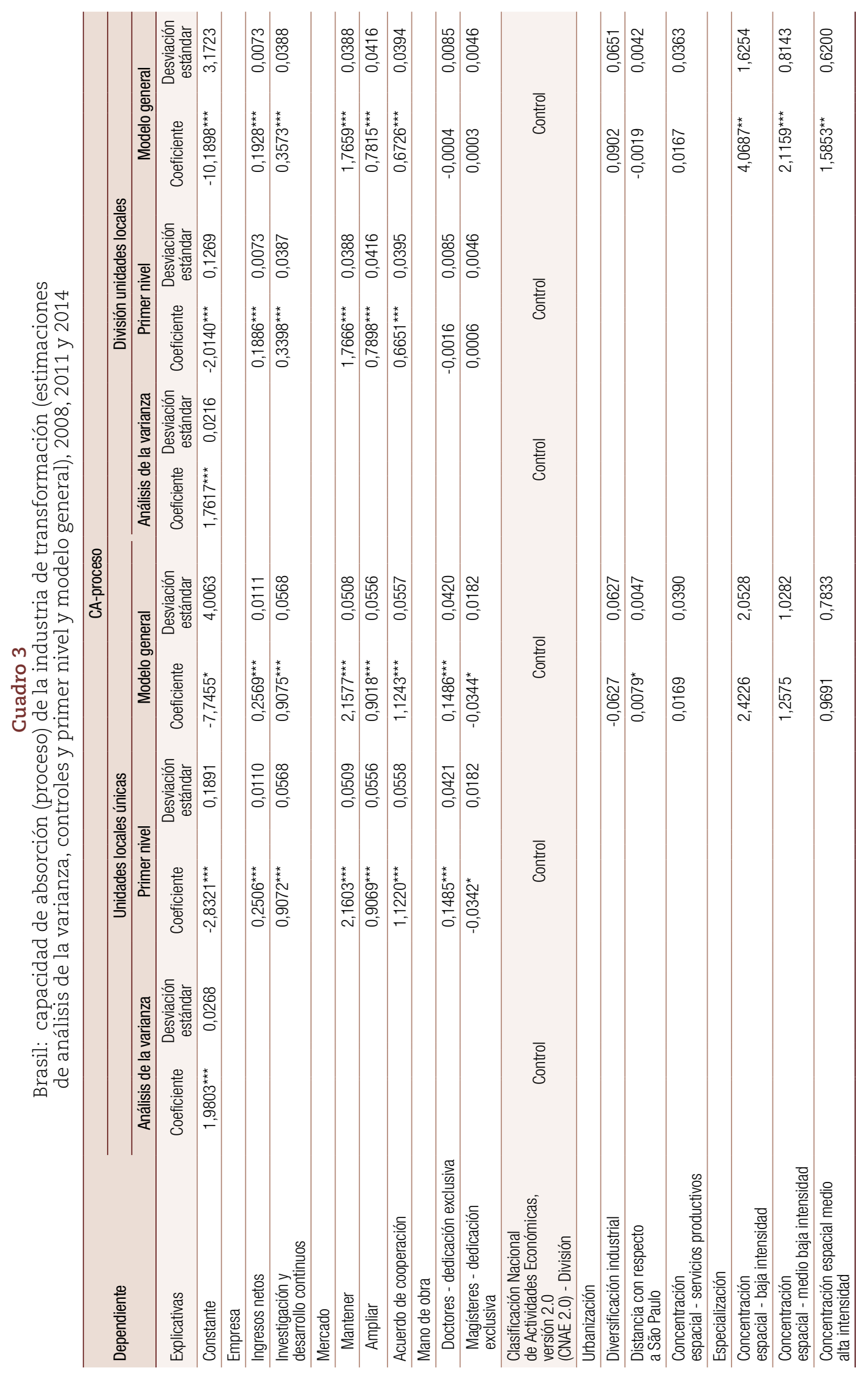




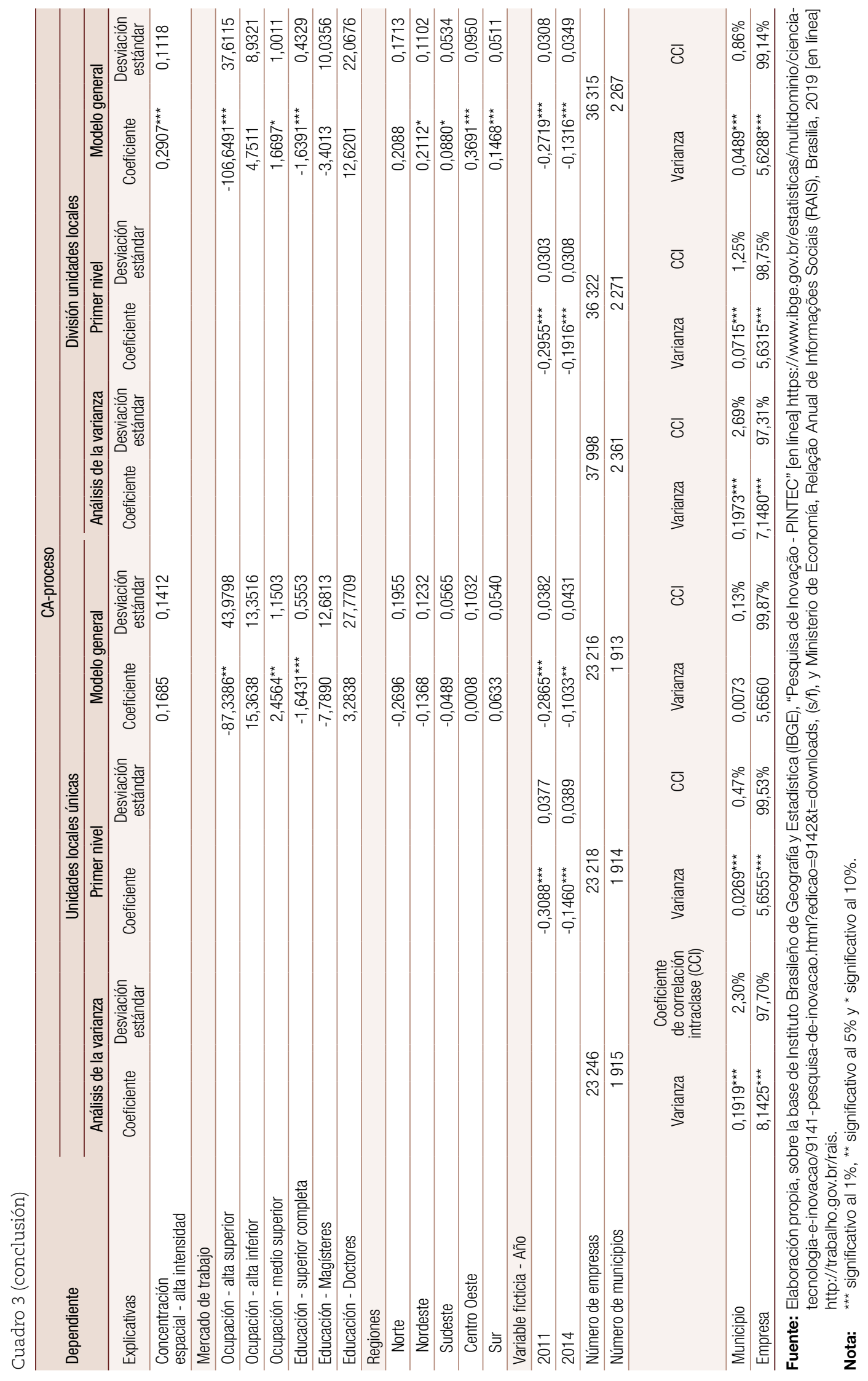


Con respecto a los controles de localización, se observan diferencias en los resultados al comparar los dos grupos de empresas ("unidades locales únicas" y "todas las empresas con valores divididos entre unidades locales"). Para el primer grupo, los controles de "especialización" no fueron significativos y, por lo tanto, no hubo mayor influencia de este "espacio" en el aumento de los gastos en "CA-proceso", como se esperaba. Un resultado esperado, también para este grupo de empresas, es el incremento de los gastos a medida que aumenta la distancia con respecto al municipio de São Paulo, lo que sugiere una relación con los factores disgregantes (Von Thünen, 1966). Cuando el análisis se extiende a toda la PINTEC, y se vuelve necesario adaptar su realidad ${ }^{15}$ a la propuesta de este artículo, los factores de especialización según la localización se muestran dentro de lo esperado conforme la teoría, con influencia de este espacio en el crecimiento de los gastos. A su vez, esta influencia crece a medida que disminuye la intensidad tecnológica sectorial. En esta segunda estimación, la distancia con respecto a São Paulo perdió significación.

Los determinantes de la empresa para los gastos en capacidad de absorción presentaron los signos y la significación esperados. La variable sustitutiva de las oportunidades tecnológicas fue la existencia de actividades continuas de investigación y desarrollo, entendiendo que las empresas localizadas en sectores económicos con grandes oportunidades necesitan estar constantemente conectadas con los avances productivos y científicos (Cohen y Levinthal, 1990; Klevorick y otros, 1995; Albuquerque, 1998; entre otros). La posibilidad de usufructuar de los resultados de sus gastos en actividades innovadoras quedó definida como condiciones de apropiabilidad (Nelson y Winter, 1982; Cohen y Levinthal, 1990; Albuquerque, 1998; entre otros). La existencia de tales condiciones reforzaría la estrategia de las empresas de invertir en innovación. La variable sustitutiva utilizada fue la definición de que los resultados del proceso innovador permitieron el mantenimiento o la ampliación de su cuota de mercado, sugiriendo la existencia de retornos favorables al proceso innovador. Una vez más, el signo observado coincidió con lo esperado. Los resultados confirmaron la influencia positiva de los acuerdos de cooperación para la innovación en el desarrollo de capacidades de absorción, ya destacada por Veja-Jurado, Gutiérrez-Gracia y Fernández-de-Lucio (2008).

\section{Conclusión}

El artículo contribuye a la literatura al medir los determinantes de la inversión en la capacidad de absorción de las empresas industriales brasileñas, teniendo en cuenta la importancia de los condicionantes territoriales en este aspecto. En general, los resultados obtenidos en los ejercicios econométricos encuentran amparo teórico, con determinantes internos de la empresa de "CA-producto" o "CA-proceso" que presentan los signos y la significación esperados.

En el caso de los condicionantes territoriales, se esperaba una mayor correlación entre la investigación y el desarrollo y los atributos urbanos (diversificación productiva, especialización en servicios productivos y proximidad con la ciudad de São Paulo), que no se confirmó (Jacobs, 1975; Duranton y Puga, 2000; Storper y Venables, 2004; entre otros). Esto puede explicarse por la fragilidad brasileña para desarrollar, de manera más eficaz y voluminosa, los gastos en estas actividades. Esto justificaría el atraso del Brasil, sugiriendo la posibilidad de que el incremento de esos gastos podría generar importantes efectos nacionales en términos de ganancias de productividad y mercados, aunque en un primer momento los avances se limitaran a alcanzar un mercado nacional.

Como se esperaba, los resultados para CA-proceso señalan a los espacios especializados como principales "destinos" de los gastos efectuados en "máquinas y equipos", "capacitación" y "software" (para los resultados en que los gastos se dividieron entre las unidades locales). Duranton y Puga (2001)

15 No desagregada a nivel municipal. 
ya habían llamado la atención sobre la posibilidad de que, a pesar de ser muy favorable para el florecimiento de actividades de investigación y desarrollo, el espacio urbano diversificado presente una serie de obstáculos a la expansión de la actividad productiva, de manera que el espacio especializado pasa a ser preferible, al ofrecer las externalidades de localización/MAR ${ }^{16}$ sin los altos costos del espacio urbano diversificado. También como se esperaba y se observó en los resultados, el aumento de la influencia de la especialización en los gastos con la disminución de la intensidad tecnológica se justifica por el hecho de que cuanto menor sea la intensidad, menores serán la complejidad productiva y los niveles de productividad. Esta menor complejidad productiva supone menos capacidad para cubrir los costos de este "espacio localizado" y, al demandar menos atributos urbanos, la producción termina situándose en los espacios especializados. Otro factor que refuerza este movimiento, y que también se observa en los ejercicios empíricos, se refiere a los costos de la mano de obra (Puga y Venables, 1996).

A pesar de que los resultados son satisfactorios desde el punto de vista de la alineación con las teorías que fundamentaron el análisis, los resultados de los atributos urbanos pueden estar influenciados por las limitaciones de la PINTEC en lo que se refiere al análisis territorial, visto que las dos estrategias implementadas para tratar de superar esas limitaciones significaron la exclusión de un grupo de empresas con más de una unidad local ${ }^{17}$ o la distribución de los gastos en actividades innovadoras entre las unidades locales de forma arbitraria, dado que no había una alternativa dentro de la PIA-UL que permitiera una mejor indicación de la localidad en que tuvo lugar el gasto. No obstante, la identificación de esos problemas, asociada a los resultados obtenidos, refuerza la importancia de avanzar en el análisis de la influencia del ambiente en que se insertan las empresas en el proceso de innovación. La PINTEC, principal base de datos nacional sobre las características del proceso innovador, presenta limitaciones a este avance y estos problemas serían evitables si en ella se indicara el municipio donde se realizaron los gastos en actividades innovadoras, entre otros datos que permitirían evaluar el proceso innovador brasileño desde una perspectiva territorial. Como se expuso, la evaluación del proceso innovador brasileño solo desde una perspectiva sectorial deja de lado importantes factores relacionados con el territorio y las externalidades derivadas de la localización.

\section{Bibliografía}

Abramovitz, M. (1986), "Catching up, forging ahead, and falling behind", The Journal of Economic History, vol. 46, $\mathrm{N}^{\circ}$ 2, junio.

Albuquerque, E. (1998), "Patentes segundo a abordagem neo-schumpeteriana: uma discussão introdutória", Revista de Economia Política, vol. 18, № 4.

Araújo, B. y M. Salerno (2015), "Padrões tecnológicos e aprendizado de exportação: o caso das firmas industriais brasileiras, 2006-2008", Produtividade no Brasil: desempenho e determinantes - Volume 2 - Determinantes, F. De Negri y L. Cavalcante (coords.), Brasilia, Agencia Brasileña de Desarrollo Industrial (ABDI)/Instituto de Investigación Económica Aplicada (IPEA).

Araújo, V. (2014), "Dimensão local da inovação no Brasil: determinantes e efeitos de proximidade", tesis de doctorado, São Paulo, Universidad de São Paulo.

Barbour, E. y A. Markusen (2007), "Regional occupational and industrial structure: does one imply the other?", International Regional Science Review, vol. 30, № 1, enero, SAGE.

Bell, M. y K. Pavitt (1997), "Technological accumulation and industrial growth: contrasts between developed and developing countries", Technology, Globalisation and Economic Performance, D. Archibugi y J. Michie (eds.), Cambridge, Cambridge University Press.

Cavalcante, L. (2014), “Classificações tecnológicas: uma sistematização”, Nota Técnica, № 17, Brasilia, Instituto de Investigación Económica Aplicada (IPEA), marzo.

\footnotetext{
${ }^{16}$ Marshall-Arrow-Romer (Combes, 2000).

17 Probablemente quedaron excluidas empresas más grandes y, por las características del proceso innovador, más innovadoras.
} 
Cavalcante, L., P. Jacinto y F. De Negri (2015), "P\&D, Inovação e produtividade na indústria brasileira", Produtividade no Brasil: desempenho e determinantes - Volume 2 - Determinantes, F. De Negri y L. Cavalcante (coords.), Brasilia, Agencia Brasileña de Desarrollo Industrial (ABDI)/Instituto de Investigación Económica Aplicada (IPEA).

Chiarini, T. (2014), "Transferência internacional da tecnologia: interpretações e reflexões: o caso brasileiro no paradigma das TICs na última década do século XX e no alvorecer do século XXI", tesis de doctorado, Campinas, Universidad Estadual de Campinas (UNICAMP).

Cohen, W. y D. Levinthal (1990), "Absorptive capacity: a new perspective on learning and innovation", Administrative Science Quarterly, vol. 35, № 1, marzo.

(1989), "Innovation and learning: the two faces of R \& D", The Economic Journal, vol. 99, № 397, septiembre.

Combes, P. (2000), "Marshall-Arrow-Romer externalities and city growth", CERAS working paper, № 99-06, enero.

De Negri, F. (2006), "Determinantes da capacidade de absorção das firmas brasileiras: qual a influência do perfil da mão-de-obra?", Tecnologia, exportação e emprego, J. De Negri, F. De Negri y D. Coelho (coords.), Brasilia, Instituto de Investigación Económica Aplicada (IPEA).

Duranton, G. y D. Puga (2005), "From sectoral to functional urban specialisation", Journal of Urban Economics, vol. $57, N^{\circ} 2$, marzo.

(2001), "Nursery cities: urban diversity, process innovation, and the life cycle of products", American Economic Review, vol. 91, № 5.

(2000), "Diversity and specialisation in cities: why, where and when does it matter?", Urban Studies, vol. 37, N 3, SAGE, marzo.

Ernst, D. y L. Kim (2002), "Global production networks, knowledge diffusion, and local capability formation", Research Policy, vol. 31, № 8-9, diciembre.

Fajnzylber, F. (1990), “Industrialización en América Latina: de la 'caja negra' al 'casillero vacío', Cuadernos de la CEPAL, Nº 60 (LC/G.1534/Rev.1-P), Santiago, Comisión Económica para América Latina y el Caribe (CEPAL), agosto.

Feldman, M. (1994), The Geography of Innovation, Boston, Kluwer Academic Publishers.

Fontes, G., R. Simões y A. Oliveira (2010), "Urban attributes and wage disparities in Brazil: a multilevel hierarchical model", Regional Studies, vol. 44, № 5.

Freitas, E. (2012), "Economias externas, atributos urbanos e produtividade: evidências a partir do nível salarial industrial das microrregiões brasileiras, 2000-2010", tesis de maestría, Belo Horizonte, Universidad Federal de Minas Gerais (UFMG).

Galinari, R., M. Lemos y P. Amaral (2006), "Retornos crescentes urbanos: a influência do espaço na diferenciação da taxa salarial no Brasil", Tecnologia, exportação e emprego, J. De Negri, F. De Negri y D. Coelho (coords.), Brasilia, Instituto de Investigación Económica Aplicada (IPEA).

Glaeser, E. y otros (1992), "Growth in cities”, Journal of Political Economy, vol. 100, № 6, diciembre.

Goldstein, H. (1995), Multilevel Statistical Models, Londres, Edward Arnold.

Henderson, J. (2003), "Marshall's scale economies", Journal of Urban Economics, vol. 53, № 1, enero.

Henderson, J., A. Kuncoro y M. Turner (1995), "Industrial development in cities", Journal of Political Economy, vol. 103, No 5 .

IBGE (Instituto Brasileño de Geografía y Estadística) (2017), "Pesquisa Industrial Anual - Empresa - PIA-Empresa" [en línea] https://sidra.ibge.gov.br/pesquisa/pia-empresa/quadros/brasil/2017.

(s/f), "Pesquisa de Inovação - PINTEC" [en línea] https://www.ibge.gov.br/estatisticas/multidominio/ ciencia-tecnologia-e-inovacao/9141-pesquisa-de-inovacao.html?edicao=9142\&t=downloads.

Jacobs, J. (1975), La economía de las ciudades, Barcelona, Ediciones Península.

Kaldor, N. (1957), "A model of economic growth", The Economic Journal, vol. 57, № 268, diciembre.

Kim, L. y R. Nelson (2005), Tecnologia, aprendizado e inovação: as experiências das economias de industrialização recente, Campinas, Universidad Estadual de Campinas (UNICAMP).

Klevorick, A. y otros (1995), "On the sources and significance of interindustry differences in technological opportunities", Research Policy, vol. 24, № 2, marzo.

Malerba, F. (1992), "Learning by firms and incremental technical change", The Economic Journal, vol. 102, $N^{\circ} 413$, julio.

Marshall, A. (1982), Princípios de Economia, vol. 1, Os Economistas, São Paulo, Abril Cultural.

McCombie, J. y P. Thirlwall (1994), Economic Growth and the Balance-of- Payments Constraint, Nueva York, St. Martin's Press.

Merhav, M. (1972), Dependencia tecnológica, monopolio y crecimiento, Buenos Aires, Ediciones Periferia. 
Messa, A. (2015), "Determinantes da produtividade na indústria brasileira", Produtividade no Brasil: desempenho e determinantes. Volume 2 - Determinantes, F. De Negri y L. Cavalcante (coords.), Brasilia, Agencia Brasileña de Desarrollo Industrial (ABDI)/Instituto de Investigación Económica Aplicada (IPEA).

Meyer-Krahmer, F. y U. Schmoch (1998), "Science-based technologies: university-industry interactions in four fields", Research Policy, vol. 27, № 8, diciembre.

Ministerio de Economía (2019), Relação Anual de Informações Sociais (RAIS), Brasilia [en línea] http://trabalho. gov.br/rais.

Nelson, R, y S. Winter (1982), An Evolutionary Theory of Economic Change, Cambridge, The Belknap Press.

Puga, D. y A. Venables (1996), "The spread of industry: spatial agglomeration in economic development", Discussion Paper, № 279, Centre for Economic Performance (CEP).

Raudenbush, S. y A. Bryk (2002), Hierarchical Linear Models: Applications and Data Analysis Methods, Thousand Oaks, SAGE Publications.

Rodrigues, E., A. de Oliveira y E. Albuquerque (2007), "Uma análise da mobilidade ocupacional no Brasil segundo o nível tecnológico das ocupações" [en línea] http://www.abep.org.br/publicacoes/index.php/anais/article/ view/1888/1846.

Rosa, A. (2013), "Capacidade absortiva de empresas que possuem interação com universidades", tesis de maestría, São Leopoldo, Universidad do Vale do Rio dos Sinos (UNISINOS).

Rosenberg, N. (1993), Dentro de la caja negra: tecnología y economía, Barcelona, La Llar del Llibre.

Schumpeter, J. (1982), A teoria do desenvolvimento econômico, Os economistas, São Paulo, Abril Cultural.

Storper, M. y A. Venables (2004), "Buzz: face-to-face contact and the urban economy", Journal of Economic Geography, vol. 4, № 4, agosto.

Tavares, M. (1998), "Auge y declinación del proceso de sustitución de importaciones en el Brasil”, Cincuenta años de pensamiento de la CEPAL: textos seleccionados, vol. 1, R. Bielschowsky (comp.), Santiago, Comisión Económica para América Latina y el Caribe (CEPAL)/Fondo de Cultura Económica (FCE).

Teece, D. y G. Pisano (1994), "The dynamic capabilities of firms: an introduction”, Industrial and Corporate Change, vol. 3, N 3, septiembre.

Teece, D., G. Pisano y A. Shuen (1997), "Dynamic capabilities and strategic management”, Strategic Management Journal, vol. 18, № 7 , agosto.

Thirlwall, P. (2019), "La restricción de la balanza de pagos como una explicación de las diferencias internacionales de la tasa de crecimiento", Investigación Económica, vol. 78, № 308.

Van den Bosch, F., H. Volberda y M. de Boer (1999), "Coevolution of firm absorptive capacity and knowledge environment: organizational forms and combinative capabilities", Organization Science, vol. 10, Nㅜ 5.

Vega-Jurado, J., A. Gutiérrez-Gracia e I. Fernández-de-Lucio (2008), "Analyzing the determinants of firm's absorptive capacity: beyond R\&D", R\&D Management, vol. $38, N^{\circ} 4$, septiembre.

Von Thünen, J. (1966), Von Thünen's Isolated State: An English Edition of Der Isolierte Staat, P. Hall (ed.), Oxford, Pergamon Press.

Zahra, S. y G. George (2002), "Absorptive capacity: a review, reconceptualization, and extension", The Academy of Management Review, vol. 27, № 2, abril. 